\title{
Usabilidade de bombas de infusão em terapia intensiva
}

\author{
Usability of infusion pumps in intensive care
}

Usabilidad de bombas de infusión en cuidados intensivos

\begin{abstract}
Marcos Soares de Lima*, Elizandra Cássia da Silva Oliveira², Marcella Di Fátima Ferreira Noya Barros $^{1}$, Regina Célia de Oliveira ${ }^{1}$.
\end{abstract}

\section{RESUMO}

Objetivo: Analisar a usabilidade de bombas de infusão em uma unidade de terapia intensiva adulto. Métodos: Estudo descritivo com abordagem quantitativa realizado em um hospital da região metropolitana do Recife, com a equipe de enfermagem que desempenha atividades assistenciais na unidade de terapia intensiva, entre os meses de agosto a dezembro de 2019. Utilizou-se um instrumento de registro de observação, confeccionado pelos pesquisadores e a tarefa consistia em o profissional de enfermagem fazer uma tarefa simples do seu dia a dia: instalar e programar na uma solução de $528.3 \mathrm{ml}$ de SF 0,9\% em 12 horas; sendo cronometrado e registrado o tempo para o cumprimento da tarefa. Resultados: $56,7 \%$ dos profissionais concluíram a tarefa, 33,3\% cumpriram a tarefa proposta com conformidade, $23,3 \%$ cumpriram com não conformidade e $43,3 \%$ não cumpriram a tarefa. 33,3\% não tiveram nenhum erro até finalizar a tarefa; $58,9 \%$ tiveram de 1 a 5 e $7,8 \%$ tiveram de 6 a 10 erros. 23,3\% referiram fazer uso da calculadora; 22,2\% relataram que não teve treinamento. Conclusão: Notou-se a baixa usabilidade da bomba de infusão e um dos fatores que possa ter contribuído foi a falta de treinamento/pouco treinamento, necessitando de estratégias de educação continuada, para capacitar/atualizar os profissionais.

Palavras-chave: Bombas de infusão, Cuidados críticos, Segurança do paciente.

\begin{abstract}
Objective: To analyze the usability of infusion pumps in an adult intensive care unit. Methods: A descriptive study with a quantitative approach carried out in a hospital in the metropolitan region of Recife, with the nursing team that performs care activities in the intensive care unit, between the months of August to December 2019. An instrument for registering patients was used. observation, made by the researchers and the task consisted of the nursing professional doing a simple daily task: installing and programming in a solution of $528.3 \mathrm{ml}$ of SF $0.9 \%$ in 12 hours; being timed and recorded the time for the accomplishment of the task. Results: $56.7 \%$ of professionals completed the task, $33.3 \%$ completed the proposed task with compliance, $23.3 \%$ completed it with non-compliance and $43.3 \%$ did not complete the task. $33.3 \%$ had no errors until the task was completed; $58.9 \%$ had 1 to 5 and $7.8 \%$ had 6 to 10 errors. $23.3 \%$ reported using the calculator; $22.2 \%$ reported that they had no training. Conclusion: The low usability of the infusion pump was noted and one of the factors that may have contributed was the lack of training / little training, requiring continuing education strategies to train / update professionals.
\end{abstract}

Key words: Infusion pumps, Critical care, Patient safety.

\section{RESUMEN}

Objetivo: Analizar la usabilidad de las bombas de infusión en una unidad de cuidados intensivos para adultos. Métodos: Estudio descriptivo con abordaje cuantitativo realizado en un hospital de la región metropolitana de Recife, con el equipo de enfermería que realiza actividades asistenciales en la unidad de cuidados intensivos, entre los meses de agosto a diciembre de 2019. Instrumento de registro de observación, realizado por los investigadores y la tarea consistió en que el profesional de enfermería realizara una sencilla tarea diaria: instalar y programar en una solución de $528,3 \mathrm{ml}$ de SF $0,9 \%$ en 12 horas; siendo cronometrado y registrado el tiempo para la realización de la tarea. Resultados: El $56,7 \%$ de los profesionales completó la tarea, el

\footnotetext{
${ }^{1}$ Universidade de Pernambuco (UPE), Recife - PE. *E-mail: marcos55soares@hotmail.com

${ }^{2}$ Hospital da Restauração Governador Paulo Guerra (HR), Recife - PE.
}

SUBMETIDO EM: 3/2021

ACEITO EM: 4/2021

PUBLICADO EM: 4/2021 
$33,3 \%$ completó la tarea propuesta con cumplimiento, el 23,3\% la completó con incumplimiento y el 43,3\% no completó la tarea. El 33,3\% no tuvo errores hasta que se completó la tarea; El 58,9\% tenía de 1 a 5 y el 7,8\% tenía de 6 a 10 errores. El 23,3\% informó usar la calculadora; El 22,2\% informó que no tenía formación. Conclusión: Se notó la baja usabilidad de la bomba de infusión y uno de los factores que pudo haber contribuido fue la falta de capacitación / poca capacitación, requiriendo estrategias de educación continua para capacitar / actualizar a los profesionales.

Palabras clave: Bombas de infusión, Cuidado crítico, Seguridad del paciente.

\section{INTRODUÇÃO}

Na Unidade de Terapia Intensiva (UTI) encontra-se um dos grandes arsenais tecnológicos e modernos essenciais aos cuidados do paciente grave (RIBEIRO GSR, et al., 2017). Dentre tantos equipamentos utilizados em UTI, se insere a Bomba de Infusão (BI) como um Equipamento Médico Assistencial (EMA) bastante utilizado; constituindo-se atualmente em um forte aliado à equipe de enfermagem, no processo de administração de fluidos, dietas e medicamentos com segurança ao paciente grave (LANGE K, et al.,2016).

A BI é um EMA que funciona por meio de pressão positiva, gerando e regulando a vasão de drogas ou dietas infundidas no paciente grave; e para que não ocorra eventos adversos, esse EMA dispõe de tela de comando e sistema de alarmes modernos, que garantem maior precisão e segurança na infusão de soluções administradas ao paciente na UTI (CALAVARO JO, 2020).

Sua principal função é a administração contínua e precisa de drogas que agem em um limiar entre sua ação terapêutica e efeito tóxico muito próximos, que quando administrados em outros EMAs, ao exemplo dos equipos simples, podem não ser tão precisos e contínuos na sua infusão, gerando assim eventos adversos graves e infusão imprecisa (CAVALARO JO, 2020). Utilizar-se de BI em pacientes críticos é de extrema importância, pois possibilita maior controle e rigor nas administrações, maior eficácia na conduta terapêutica do paciente e garantia de segurança na administração de medicamentos e dietas (HOLSBACH LR, 2013).

Ao utilizar um EMA a interação humano-equipamento é importante; onde o usuário precisa configurar o equipamento, a fim de garantir a efetividade e eficácia de todas as suas funções (LANGE K, et al., 2016). Esta interação pode ser avaliada através da usabilidade do equipamento que abrange a forma como este produto é manuseado por profissionais específicos, para atingir metas específicas, em um contexto peculiar de uso; compreendido como um equipamento fácil de usar e de aprender na prática assistencial (SCHMETTOW M, et al., 2017).

Embora a $\mathrm{BI}$ seja considerado um equipamento fácil de manusear; problemas relacionados à sua usabilidade têm sido apontados, como dispositivos e software antiquados que diferem das necessidades dos usuários e a complexidade de medicamentos e fluidos selecionados e tamanho reduzido da tela (ROQUE KE, et al., 2016; SCHNITTKER R, et al., 2016). Assim, a Food and Drug Administration (FDA) evidenciou que a complexidade operacional das bombas de infusão levou a 56.000 eventos adversos de medicamentos durante um período de quatro anos, alguns dos quais resultaram em eventos graves e mortes (PHAM JC, et al., 2016).

Tendo em vista o contexto vivenciado e a importância da segurança do paciente e o manuseio correto da $\mathrm{BI}$; percebe-se lacunas científicas que abordem a usabilidade deste equipamento em UTI adulto. Sendo pertinente o seguinte questionamento: o manuseio da $\mathrm{BI}$ pela equipe de enfermagem em terapia intensiva adulto vem sendo realizado de forma segura? O objetivo deste estudo é analisar a usabilidade de bombas de infusão em uma unidade de terapia intensiva adulto.

\section{MÉTODOS}

Trata-se de um estudo descritivo com uma abordagem quantitativa, realizado na unidade de terapia intensiva adulto de um hospital da região metropolitana do Recife. O estudo é parte integrante da construção de uma Tese de Doutorado intitulada: Impacto da usabilidade da bomba de infusão no tempo estímulo resposta da fadiga de seus alarmes, associada ao programa de Pós-graduação em Enfermagem da Universidade de Pernambuco e Universidade estadual da paraíba (UPE/UEPB). 
A população de estudo foi composta pela equipe de enfermagem (Enfermeiros e técnicos de enfermagem) que desempenha atividades assistenciais e manuseia a bomba de infusão na unidade de terapia intensiva adulto. Sendo a amostra censitária. Os critérios de inclusão adotados foram: profissionais de enfermagem inseridos em atividades de assistência direta ao paciente e manuseiam a $\mathrm{Bl}$; e os critérios de inclusão: profissionais de enfermagem que trabalham em atividades indiretas (coordenação de enfermagem) na unidade de terapia intensiva e não manuseiam a BI.

A coleta de dados foi iniciada após a aprovação pelo Comitê de Ética em Pesquisa envolvendo Seres Humanos do complexo hospitalar da Universidade de Pernambuco entre os meses de agosto a dezembro de 2019, nos turnos da tarde e noite em dois dias na semana. O equipamento utilizado foi a bomba de infusão volumétrica, apropriada para infusão de soluções tanto por via enteral, quanto por via parenteral, incorporando todos os requisitos de segurança estabelecidos na norma NBR IEC 60601-2-24. Para a coleta de dados, foi utilizado um instrumento de registro de observação - pesquisa de campo, confeccionado com base nas normas de uso estabelecida pelo manual do fabricante da bomba de infusão no tópico: modo de operar a BI.

A tarefa avaliada consistia em o profissional de enfermagem fazer uma tarefa simples do seu dia a dia: instalar e programar na BI uma solução de $528.3 \mathrm{ml}$ de SF $0,9 \%$ em $12 \mathrm{hs}$; e a partir disso podia ser classificada em: "Em conformidade" - quando o cumprimento do passo a passo era realizado sem erros, obtendo o resultado correto da vazão; "Tarefa cumprida com não conformidade" — quando o cumprimento do passo a passo era realizado com erros e ainda assim, o resultado correto da vazão era obtido; e "Tarefa não cumprida" - quando, ao seu final, o resultado da vazão era diferente do esperado. Sendo ainda cronometrado e registrado o tempo dispendido para o cumprimento da tarefa.

Os dados foram inseridos em uma planilha do EXCEL e o programa utilizado para obtenção dos cálculos estatísticos foi o Statistical Package for the Social Sciences (SPSS) na versão 25. Analisou-se descritivamente através de frequências absolutas e percentuais para as variáveis categóricas. Para avaliar associação entre duas variáveis categóricas foi utilizado o teste Qui-quadrado de Pearson ou o teste Exato de Fisher quando a condição para utilização do teste Qui-quadrado não foi verificada. O nível de significância utilizado na decisão dos testes estatísticos foi de $5 \%$.

O estudo foi aprovado pelo comitê de ética em pesquisa do Complexo Hospitalar da Universidade de Pernambuco, sob o número 2.835.374, no ano de 2018. Seguiu-se as Diretrizes e Normas Regulamentadoras de Pesquisa Envolvendo Seres Humanos, através da Resolução 466/2012 do Conselho Nacional de Saúde. Os dados coletados foram utilizados como base para a pesquisa, sendo essas informações adquiridas após o consentimento dos sujeitos entrevistados. Todos os interlocutores que participaram ao estudo, tiveram o Termo de Consentimento Livre e Esclarecido (TCLE) assinado e entregado uma das vias para os mesmos. Foi explicado aos participantes o objetivo do estudo, a finalidade e o caráter sigiloso, sendo sua participação completamente voluntária e não houve custo para os mesmos, deixando-os cientes que poderiam deixar de participar da pesquisa a qualquer momento, sem que os mesmos fossem prejudicados.

\section{RESULTADOS}

Quanto a usabilidade da bomba de infusão pela equipe de enfermagem na terapia intensiva adulto; observa-se na Tabela 1 que dos 90 profissionais de enfermagem, 75,6\% eram técnicos de enfermagem e $24,4 \%$ eram enfermeiros. Referente ao tempo que os profissionais trabalham com o tipo de $\mathrm{BI}$ estudada, prevaleceu o tempo de 1 a 5 ano em $52,2 \%$ e de até 1 ano em $32,2 \%$ dos profissionais trabalham com este modelo de BI (Tabela 1). Em relação a operacionalização da bomba de infusão, verificou-se que $56,7 \%$ dos profissionais concluíram a tarefa, sendo que destes $33,3 \%$ cumpriram a tarefa proposta com conformidade $23,3 \%$, cumpriram a tarefa com não conformidade e $43,3 \%$ não cumpriram a tarefa. No quesito número de erros para completar a tarefa, foi prevalente em $58,9 \%$ o total de 1 a 5 erros.

Quando se analisa o tempo que cada profissional levou para cumprir ou passou tentando cumprir a tarefa evidencia-se que prevaleceram os tempos de $>0: 30$ a 1:00 minuto em 35,6\% e 26,7\% de $>1: 00$ a 1:30 minutos. Para concluir a tarefa proposta o participante teria que fazer 10 comandos na $\mathrm{BI}$ até obter o resultado correto da vazão a ser infundida no paciente; tendo em vista esses comandos, obteve-se os resultados a 
seguir: $100 \%$ pressionaram a tecla liga/desliga, $84,4 \%$ aguardaram a rotina e o auto teste, $98,9 \%$ pressionaram a tecla $\mathrm{F} 1,97,8 \%$ pressionaram a tecla $2,86,7 \%$ souberam programar o volume de infusão para 528,3 $\mathrm{ml}$ de SF 0,9\%, 62,2\% pressionaram a tecla F2, 54,4\% pressionaram a tecla 4, 40\% conseguiram programar o tempo de infusão para 12 horas, 37,8\%pressionaram a tecla $\mathrm{F} 3$ e por último apenas 56,7\% chegaram a clicar na tecla Start e concluir a tarefa com, sendo está com ou sem conformidade. Ao final de cada entrevista os profissionais ficaram livres para falar o que achou da entrevista, dizer alguma dificuldade com o manuseio da bomba ou na atividade proposta, e chama atenção o fato de $23,3 \%$ referirem fazer uso da calculadora para realizar o manuseio e administrar as medicações ou dieta no paciente; $22,2 \%$ relatarem que não teve treinamento com esse modelo de $\mathrm{BI}$ e 11,1\% que declaram não saber manusear a $\mathrm{BI}$.

Tabela 1 - Características da equipe de enfermagem e operacionalização da bomba de infusão em unidade de terapia intensiva adulto.

\begin{tabular}{|c|c|c|}
\hline Variável & $\mathbf{n}$ & $\%$ \\
\hline \multicolumn{3}{|l|}{ Profissão } \\
\hline Técnico de enfermagem & 68 & 75,6 \\
\hline Enfermeiro & 22 & 24,4 \\
\hline \multicolumn{3}{|c|}{ Tempo que trabalha com este tipo de BI (anos) } \\
\hline Até 1 & 29 & 32,2 \\
\hline$>1$ a 5 & 47 & 52,2 \\
\hline$>5$ a 10 & 11 & 12,2 \\
\hline$>10$ a 15 & 1 & 1,1 \\
\hline$>15$ & 2 & 2,2 \\
\hline \multicolumn{3}{|l|}{ Tarefa concluída } \\
\hline Sim & 51 & 56,7 \\
\hline Não & 39 & 43,3 \\
\hline \multicolumn{3}{|l|}{ Número de erros } \\
\hline Nenhum & 30 & 33,3 \\
\hline 1 a 5 & 53 & 58,9 \\
\hline 6 a 10 & 7 & 7,8 \\
\hline \multicolumn{3}{|l|}{ Tempo para cumprir a tarefa } \\
\hline $0: 00$ a $0: 30$ segundos & 9 & 10,0 \\
\hline$>0: 30$ segundos a $1: 00$ minuto & 32 & 35,6 \\
\hline$>1: 00$ a $1: 30$ minutos & 24 & 26,7 \\
\hline$>1: 30$ a $2: 00$ minutos & 11 & 12,2 \\
\hline$>2: 00$ minutos & 14 & 15,6 \\
\hline Pressionar tecla liga/desliga & 90 & 100,0 \\
\hline Aguarda rotina e auto-teste & 76 & 84,4 \\
\hline Pressionar tecla F1 & 89 & 98,9 \\
\hline Pressionar tecla 2 & 88 & 97,8 \\
\hline Programar o volume de infusão $(528,3)$ & 78 & 86,7 \\
\hline Pressionar tecla F2 & 56 & 62,2 \\
\hline Pressionar tecla 4 & 49 & 54,4 \\
\hline Programar tempo de infusão de $12 \mathrm{~h}$ & 36 & 40,0 \\
\hline Pressionar tecla F3 & 34 & 37,8 \\
\hline Start & 51 & 56,7 \\
\hline \multicolumn{3}{|l|}{ Depoimentos $^{(1)}$} \\
\hline Usa calculadora & 21 & 23,3 \\
\hline Não teve treinamento & 20 & 22,2 \\
\hline Não sabe fazer & 10 & 11,1 \\
\hline Não informado & 46 & 51,1 \\
\hline \multicolumn{3}{|l|}{ Classificação da tarefa } \\
\hline Em conformidade & 30 & 33,3 \\
\hline Cumprida com não conformidade & 21 & 23,3 \\
\hline Não cumprida & 39 & 43,3 \\
\hline Total & 90 & 100,0 \\
\hline
\end{tabular}

Legenda: (1) Considerando que alguns profissionais deram mais de um depoimento. Fonte: Lima MS, et al., 2020.

Observa-se que quanto eficácia e efetividade da usabilidade da BI não foi observado significância estatística em relação as tarefas propostas com o tempo em anos de trabalho com a bomba de infusão. Entretanto, 
observa-se que entre os profissionais que trabalham com esse modelo de $\mathrm{Bl}$ em até 1 ano e de $>1$ a 5 anos houve maior cumprimento da tarefa e que com o passar dos anos $(>5)$ houve uma redução no cumprimento da tarefa. Como também o número de erros para cumprir a tarefa foi maior à medida que o tempo em anos de trabalho aumentava (Tabela 2). Quanto ao tempo para realizar a tarefa foi verificado maior tempo naqueles profissionais com menor tempo de trabalho com a $\mathrm{BI}>1$ a 5 anos. Outro dado que merece destaque é o comando "aguardar a rotina de auto-teste", onde evidenciou-se que cerca de 15,6\% não esperaram a rotina de auto-teste da $\mathrm{BI}$, o que pode favorecer a ocorrência de eventos adversos.

Tabela 2 - Eficácia e efetividade da usabilidade da bomba de infusão pela equipe de enfermagem em unidade de terapia intensiva adulto.

\begin{tabular}{|c|c|c|c|c|c|c|c|c|c|}
\hline \multirow{3}{*}{ Variável } & \multicolumn{8}{|c|}{ Tempo de trabalho com BI (anos) } & \multirow{3}{*}{ Valor de $p$} \\
\hline & \multicolumn{2}{|c|}{ Até 1} & \multicolumn{2}{|c|}{$>1$ a 5} & \multicolumn{2}{|c|}{$>5$} & \multicolumn{2}{|c|}{ Grupo Total } & \\
\hline & $\mathbf{N}$ & $\%$ & $\mathbf{N}$ & $\%$ & $\mathbf{N}$ & $\%$ & $\mathbf{N}$ & $\%$ & \\
\hline Tarefa concluída & & & & & & & & & $\mathrm{p}^{(1)}=0,494$ \\
\hline Sim & 19 & 65,5 & 25 & 53,2 & 7 & 50,0 & 51 & 56,7 & \\
\hline Não & 10 & 34,5 & 22 & 46,8 & 7 & 50,0 & 39 & 43,3 & \\
\hline Número de erros & & & & & & & & & $\mathrm{p}^{(2)}=0,693$ \\
\hline Nenhum & 12 & 41,4 & 13 & 27,7 & 5 & 35,7 & 30 & 33,3 & \\
\hline 1 a 5 & 16 & 55,2 & 29 & 61,7 & 8 & 57,1 & 53 & 58,9 & \\
\hline 6 a 10 & 1 & 3,4 & 5 & 10,6 & 1 & 7,1 & 7 & 7,8 & \\
\hline Tempo para cumprir a tarefa & & & & & & & & & $\mathrm{p}^{(2)}=0,981$ \\
\hline $0: 00$ a $0: 30$ segundos & 3 & 10,3 & 5 & 10,6 & 1 & 7,1 & 9 & 10,0 & \\
\hline$>0: 30$ segundos a $1: 00$ minuto & 9 & 31,0 & 18 & 38,3 & 5 & 35,7 & 32 & 35,6 & \\
\hline$>1: 00$ a $1: 30$ minutos & 9 & 31,0 & 10 & 21,3 & 5 & 35,7 & 24 & 26,7 & \\
\hline$>1: 30$ a $2: 00$ minutos & 3 & 10,3 & 7 & 14,9 & 1 & 7,1 & 11 & 12,2 & \\
\hline$>2: 00$ minutos & 5 & 17,2 & 7 & 14,9 & 2 & 14,3 & 14 & 15,6 & \\
\hline Aguarda a rotina de auto-teste & & & & & & & & & $\mathrm{p}^{(2)}=0,789$ \\
\hline Sim & 24 & 82,8 & 39 & 83,0 & 13 & 92,9 & 76 & 84,4 & \\
\hline Não & 5 & 17,2 & 8 & 17,0 & 1 & 7,1 & 14 & 15,6 & \\
\hline $\begin{array}{l}\text { Programar o volume de infusão } \\
(528,3)\end{array}$ & & & & & & & & & $=0,915$ \\
\hline Sim & 26 & 89,7 & 40 & 85,1 & 12 & 85,7 & 78 & 86,7 & \\
\hline Não & 3 & 10,3 & 7 & 14,9 & 2 & 14,3 & 12 & 13,3 & \\
\hline Pressionar tecla F2 & & & & & & & & & $\mathrm{p}^{(1)}=0,181$ \\
\hline Sim & 21 & 72,4 & 25 & 53,2 & 10 & 71,4 & 56 & 62,2 & \\
\hline Não & 8 & 27,6 & 22 & 46,8 & 4 & 28,6 & 34 & 37,8 & \\
\hline Pressionar tecla 4 & & & & & & & & & $\mathrm{p}^{(1)}=0,312$ \\
\hline Sim & 18 & 62,1 & 22 & 46,8 & 9 & 64,3 & 49 & 54,4 & \\
\hline Não & 11 & 37,9 & 25 & 53,2 & 5 & 35,7 & 41 & 45,6 & \\
\hline $\begin{array}{l}\text { Programar tempo de infusão de } \\
12 \mathrm{~h}\end{array}$ & & & & & & & & & 60 \\
\hline Sim & 14 & 48,3 & 15 & 31,9 & 7 & 50,0 & 36 & 40,0 & \\
\hline Não & 15 & 51,7 & 32 & 68,1 & 7 & 50,0 & 54 & 60,0 & \\
\hline Press & & & & & & & & & $\mathrm{p}^{(1)}=0,055$ \\
\hline Sim & 16 & 55,2 & 13 & 27,7 & 5 & 35,7 & 34 & 37,8 & \\
\hline Não & 13 & 44,8 & 34 & 72,3 & 9 & 64,3 & 56 & 62,2 & \\
\hline Start & & & & & & & & & $\mathrm{p}^{(1)}=0,494$ \\
\hline Sim & 19 & 65,5 & 25 & 53,2 & 7 & 50,0 & 51 & 56,7 & \\
\hline Não & 10 & 34,5 & 22 & 46,8 & 7 & 50,0 & 39 & 43,3 & \\
\hline Class & & & & & & & & & $\mathrm{p}^{(2)}=0,674$ \\
\hline Confor & 12 & 41,4 & 13 & 27,7 & 5 & 35,7 & 30 & 33,3 & \\
\hline n não con & 7 & 24,1 & 12 & 25,5 & 2 & 14,3 & 21 & 23,3 & \\
\hline Não cu & 10 & 34,5 & 22 & 46,8 & 7 & 50,0 & 39 & 43,3 & \\
\hline Total & 29 & 100,0 & 47 & 100,0 & 14 & 100,0 & 90 & 100,0 & - \\
\hline
\end{tabular}

Quanto a operacionalização da BI a partir do comando "programar tempo de infusão de $12 \mathrm{~h}$ " identifica-se um percentual menor de acertos, onde apenas $40 \%$ conseguiram programar o tempo de infusão para 12 horas, $37,7 \%$ pressionaram a tecla F3 e por último apenas $56,6 \%$ chegaram a clicar na tecla Start 
corretamente. Já em relação a classificação da tarefa notou-se que profissionais com $>1$ a 5 anos de tempo de trabalho com o modelo de BI proposto tem maiores chances de não cumprir a tarefa com conformidade. No que tange aos depoimentos, apesar de não se observar significância estatística, o percentual de não treinamentos $(22,2 \%)$, não sabe fazer $(11,1 \%)$ e usar calculadora $(23,3 \%)$ são preocupantes.

\section{DISCUSSÃO}

Quando se discute o dimensionamento da equipe de Enfermagem e de recursos humanos em enfermagem para garantia na qualidade do cuidado prestado, satisfação do cliente e a quantidade de horas de assistência ao paciente, sabe-se que a relação de enfermeiros para técnicos de enfermagem por leito varia conforme o nível de complexidade no atendimento a ser prestado (VITURI DW, et al., 2011). Sabe-se que a prestação de cuidado em UTI é caracterizada por inúmeras atividades complexas que exigem alta competência e habilidade técnica e científica, além da rápida tomada de decisões e adoção de condutas seguras, tendo em vista que a correta prestação desse cuidado está diretamente relacionada à vida e à morte dos pacientes (INOUE KC e MATSUDA LM, 2010).

Dessa forma, devido a UTI se tratar de um ambiente com tamanha complexidade, e necessitar de profissionais de enfermagem altamente qualificados e adequadamente dimensionados para desenvolver a assistência de enfermagem com qualidade e segurança, a Resolução da Diretoria Colegiada (RDC) $n^{\circ} 7$ de 2010, que dispõe sobre os requisitos mínimos para funcionamento de UTI define que para que sejam prestados os cuidados necessários aos pacientes de forma ampliada em UTI é necessário um enfermeiro para cada 8 leitos e 1 técnico de enfermagem para cada dois 2 leitos, além de 1 técnico de enfermagem por UTI para serviços de apoio assistencial em cada turno (COFEN, 2010).

Destaca-se que a UTI em estudo possui um bom dimensionamento da equipe de enfermagem, sendo verificado um quantitativo ideal para assistência ao paciente em cuidados críticos, observando-se 22 enfermeiros, sendo um por UTI em cada turno; e 68 técnicos de enfermagem. Ressalta-se que havia mais enfermeiros e mais técnicos de enfermagem, porém não foi possível fazer a coleta com todos.

Dentre todo esse aparato tecnológico que estão inseridos e utilizados em UTI, destaca-se a bomba de infusão (BI), que é um equipamento simples, mas de alta complexidade, sendo responsável por proporcionar maior precisão na administração de infusões intravenosas (sedação, drogas vasoativas, anticoagulantes e outros) ou dietas, utilizadas especialmente em pacientes que estão em estado crítico e instáveis que exigem uma maior precisão na dosagem de medicações e/ou dietas, pois qualquer erro pode resultar em eventos adversos fatais (MOREIRA APA, et al., 2020; BRASIL, 2014).

Destaca-se que no Brasil, problemas relacionados ao manuseio da BI são responsáveis por cerca de $35 \%$ dos erros de medicação realizado pela equipe de enfermagem (CHIANCA TCM e TANNURE MC, 2015; DUARTE, SCM. et al.,2015) e segundo dados do Institute of Medicine, na publicação "To Err is Human: Building a Safer Health System" os eventos adversos são a quarta causa mais importante de óbito nos Estados Unidos da América (PRATES DO e SILVA AEBC, 2016).

Dessa forma é extremamente necessário que as equipes de enfermagem que atuam no processo de cuidar nas UTIs, estejam cada vez mais capacitadas e atualizadas para operacionalizar eficaz e efetivamente em tempo oportuno este equipamento, sendo o ideal que apenas enfermeiros e técnicos de enfermagem treinados manuseiem a BI (OLIVEIRA FA e PAES GO, 2020).

Referente ao cumprimento da tarefa solicitada identificou-se que grande maioria dos profissionais trabalham com o modelo de $\mathrm{Bl}$ em estudo em um tempo de até 1 ano e de $>1$ a 5 anos, e entre estes houve maior cumprimento da tarefa proposta, porém com o passar dos anos $(>5)$ verificou-se que houve uma redução no cumprimento da tarefa.

Pode-se associar esses dados a questão das atualizações dos equipamentos que acontecem no decorrer dos anos, assim como a constante inserção de novos equipamentos nos serviços, o que requer atualizações frequentes dos profissionais; e as condições que envolvem a prestação de cuidados de enfermagem (muito paciente para poucos profissionais, carga horaria de trabalho extensa) que podem levar por vezes, à 
ocorrência de avaliações, decisões e práticas menos corretas não intencionais por parte dos enfermeiros e técnicos de enfermagem, que podem originar erros/eventos adversos ao paciente, sendo reduzida a eficiência e efetividade nas atividades (PRATAS AR, 2017).

Enfatiza-se que apesar de $56,7 \%$ dos profissionais cumprirem a tarefa, grande parte $23,3 \%$, não soube gerir o equipamento com conformidade. Esses dados constatam que no fator eficiência e eficácia há uma carência e os profissionais de Enfermagem dessa unidade referem falta de treinamento como o fator principal para o desempenho adequado das técnicas, além de utilizarem de soluções alternativas, tendo como consequência o evento adverso e não uso dos benefícios dos EMAs em sua totalidade (RIBEIRO GSR, et al., 2018).

As soluções alternativas se inserem quando os prestadores de cuidados de saúde percebem que o uso de normas e padronizações afetam negativamente suas práticas devido a incompatibilidades de fluxo de trabalho. Estas soluções alternativas são definidas como "práticas temporárias informais para lidar com exceções ao fluxo de trabalho normal," que "não seguem regras explícitas ou implícitas, suposições, regulamentos de fluxo de trabalho ou intenções de designers de sistemas"; elas permitem que os profissionais prossigam desenvolvendo suas tarefas, em especial quando sob condições de alta pressão de tempo estão esgotados e sobrecarregados com múltiplas atividades assistenciais (BLIJLEVEN V, et al., 2017).

Associa-se estas práticas de soluções alternativas, especialmente a ineficácia e não efetividade do serviço exercido, e por consequência maiores chances de ocasionar erro (BLIJLEVEN V, et al., 2017). Destaca-se como uma solução alternativa encontrada o uso constante da calculadora, que foi verificado em $23,3 \%$ dos profissionais.

Elenca-se um dado que se mostrou bem importante na análise dos dados obtidos, que foi a quantitativo de profissionais que não cumpriram a tarefa. No total $43,3 \%$ dos profissionais não concluíram e cometeram algum erro no decorrer da atividade, o que é um fator grave para segurança do paciente. Não cumprir a tarefa pode trazer dois problemas, primeiro é o não recebimento da droga e piora do quadro clínico, e o segundo é a infusão dessa droga, porém com erro e ocasionamento de evento adverso grave ao paciente que já se encontra instável (SANDRA L, et al., 2017).

Nesse sentido à para prevenção desses eventos adverso a UTI deve incorporar estratégias em todas as fases do processo de uso e administração de medicamentos (prescrição, dispensação, administração, monitoramento), dentre estas insere-se: capacitação dos profissionais, monitoramento das infusões medicamentosas, redução da exaustão do profissionais e uso de bombas de infusão com programações mais objetivas, sendo estas estratégias bastante promissoras para contornar melhorar os resultados dos pacientes (NASCIMENTO KMB, et al., 2020).

Considerando que o gerenciamento da $\mathrm{BI}$ é de grande relevância para a segurança do paciente, é necessário fomentar a importância do manuseio adequado entre os profissionais durante o processo de cuidar e fortalecer na prática o treinamento, com a educação continuada. Tem-se a educação continuada como um conjunto de atividades para atualização profissional, além de oportunizar o desenvolvimento continuado dos trabalhadores em saúde trazendo o desenvolvimento continuado do profissional no seu ambiente de trabalho (RIBEIRO BCO, et al., 2019). A educação continuada em saúde é elaborada de maneira coletiva, buscando vivências para novos instrumentos de trabalho, e desse modo potencializar a capacitação dos profissionais através das necessidades de cada serviço/setor (RIBEIRO BCO, et al., 2019). No contexto da equipe de enfermagem do estudo, percebe-se a carência de educação continuada, sendo isso percebido através dos seguintes dados: $22,2 \%$ dos profissionais não tiveram treinamento, $43,3 \%$ não soube fazer a tarefa e tiverem erros $23,3 \%$ buscaram alternativas não usuais para cumprir a tarefa.

\section{CONCLUSÃO}

Ao avaliar a usabilidade da BI identificou-se que embora ela seja um EMA de grande importância e teor tecnológico, nota-se que há alguns problemas relacionados à sua usabilidade; sendo um dos fatores contribuintes a ausência de treinamento/pouco treinamento; o que acarreta a falta de autoconfiança dos 
profissionais de enfermagem, que negam o uso da $\mathrm{BI}$ e suas atividades que são bem práticas, quando bem manuseadas. Esperasse que pesquisas voltadas à temática e que os desenvolvedores de $\mathrm{Bl}$ avaliem a usabilidade e desenvolvam modelos menos complexos, assim como também, crie, junto com os hospitais, através da educação continuada, estratégias para capacitar/atualizar os profissionais de enfermagem que manuseiam essas $\mathrm{BI}$.

\section{REFERÊNCIAS}

1. BLIJLEVEN V, et al. Work arounds Emerging from Electronic Health Record System Usage: Consequences for Patient Safety, Effectiveness of Care, and Efficiency of Care. JMIR Hum Factors, 2017; 4(4): e27.

2. BRASIL. Ministério da saúde. 2014. Documento de referência para o Programa Nacional de Segurança do Paciente. Fundação Oswaldo Cruz. Disponível em: http://bvsms.saude.gov.br/bvs/publicacoes/documento_referencia_programa_nacional_seguranca.pdf. Acesso em: 08 out. 2020.

3. CAVALARO JO, et al. Uso da Bomba de infusão em Terapia Intensiva: perspectivas da equipe de enfermagem. Rev Enferm UFSM, 2020; 32(10): 1-18.

4. CHIANCA TCM, TANNURE MCA. Tecnologia como instrumento facilitador do cuidado integral de enfermagem. Rev. enfermagem revista, 2015;17(2):1074-1081.

5. COFEN. Conselho Federal de Enfermagem. 2010. Resolução - RDC N 7 Determina Redução no Número de Leitos Por Enfermeiro Em UTIS. Disponível em:http://www.cofen.gov.br/resoluo-rdc-n-7-determina-reduo-no-numero-deleitos-por-enfermeiro-em-utis_4566.html. Acesso em: 08 out. 2020.

6. DUARTE SCM, et al. O erro humano no cotidiano da assistência de enfermagem em terapia intensiva. Rev. latinoam. Enfermagem, 2015; 23(6): 1074-1081.

7. HOLSBACH LR, et al. Utilização do instrumento de identificação de conhecimentos para administração segura de medicamentos com o uso de infusão automática. Rev Bras Eng Bioméd, 2013; 29(4):353-62.

8. INOUE KC, MATSUDA LM. Dimensionamento de pessoal de enfermagem em Unidade de Terapia Intensiva para adultos. Acta Paul. Enferm. 2010; 23(3): 379-384.

9. LANGE K, et al. A human factors perspective on medical device alarms: Problems with operating alarming devices and responding to device alarms. Biomed izinische Technik, 2016; 61(2): 147-164.

10. MOREIRA APA, et al. Handling errors in conventional and smart pump infusions: A systematic review with metaanalysis. Rev Esc Enferm USP. 2020; 54: e03562.

11. NASCIMENTO KMB, et al. Predisposition to adverse events in an Intensive Care Unit. Research, Society and Development, 2020; 9(8): e829986627.

12. OLIVEIRA FA, PAES GO. Segurança do paciente na usabilidade de bombas de infusão na terapia intensiva: revisão integrativa. Saúde Coletiva (Barueri), 2020; 52(10): 2192-2209.

13. PHAM, JC, et al. Comparison of automated versus manual programming of infusion pumps. Biomedical Instrumentation and Technology, 2016; 50(4): 242-251.

14. PRATAS ARPA. Fatores que influenciam a ocorrência de erros de medicação, enquanto está sob gestão do enfermeiro, no Serviço de Urgência. Escola Superior de Enfermagem de Coimbra, 2017.

15. PRATES DO, SILVA AEBC. Interrupções de atividades vivenciadas por profissionais de enfermagem em unidade de terapia intensiva. Rev. Latino-Am. Enfermagem, 2016; 24:2802.

16. RIBEIRO BCO, et al. A importância da educação continuada e educação permanente em unidade de terapia intensiva-revisão de literatura. Rev Inic Cient Ext., 2019; 2(3): 167-75.

17. RIBEIRO GSR, et al. Falhas de equipamentos: condutas dos enfermeiros e implicações na segurança do paciente. Rev. Bras. Enferm. Brasília, 2018; 71(4): 1832-1840.

18. RIBEIRO GSR, et al. Violações no uso de equipamentos por enfermeiros na terapia intensiva. Texto Contexto Enferm, 2017; 26(2): 6050015.

19. ROQUE KE, et al. Eventos adversos na unidade de terapia intensiva: impacto na mortalidade e no tempo de internação em um estudo prospectivo. Cad. Saúde Pública, 2016; 32(10): 1-15.

20. SANDRA L, et al. Diretriz de prática clínica: uso seguro de medicamentos na UTI. Ccm jornal, 2017; 45(9): 1-39.

21. SCHMETTOW M, et al. An extended protocol for usability validation of medical devices: Research design and reference model. J Biomed Inform, 2017; 69: 99-114.

22. SCHNITTKER R, et al. Combining situated Cognitive Engineering with a novel testing method in a case study comparing two infusion pump interfaces. Applied Ergonomics, 2016; 55: 16-26.

23. VITURI DW, et al. Dimensionamento de enfermagem hospitalar: modelo OPAS/OMS. Texto Contexto Enferm, 2011; 20(3): 547-556. 